Itinéraires Itinéraires

Littérature, textes, cultures

2010-2 | 2010

Les blogs

\title{
Itinéraire d'un blogueur : entre quête de reconnaissance et visibilité limitée
}

Olivier Trédan

\section{(2) OpenEdition}

Journals

Édition électronique

URL : http://journals.openedition.org/itineraires/1989

DOI : 10.4000/itineraires.1989

ISSN : 2427-920X

Éditeur

Pléiade

Édition imprimée

Date de publication : 1 juillet 2010

Pagination : 83-93

ISBN : 978-2-296-12012-9

ISSN : 2100-1340

Référence électronique

Olivier Trédan, «Itinéraire d'un blogueur : entre quête de reconnaissance et visibilité limitée », Itinéraires [En ligne], 2010-2 | 2010, mis en ligne le 01 juillet 2010, consulté le 21 avril 2019. URL : http:// journals.openedition.org/itineraires/1989; DOI : 10.4000/itineraires.1989

\section{(ब) $(\Theta \Theta$}

Itinéraires est mis à disposition selon les termes de la licence Creative Commons Attribution - Pas d'Utilisation Commerciale - Pas de Modification 4.0 International. 


\title{
Itinéraire d'un blogueur : entre quête de reconnaissance et visibilité limitée
}

\begin{abstract}
Blogs are known to have facilitated the access of Internet users to online publication. Nevertheless, behind this comparatively technical simplicity, the practice of blogging appears to be fraught with many traps. Through the trajectory of a blogger, our intention is to illustrate those difficulties and to show up the springs of a practice. That trajectory can be revealed in the light of a tension, inherent to the activity of publication, between peculiar appearances and a recognition on the part of peers by supporting their own world in a dynamics of permanent expansion.
\end{abstract}

Keywords : blogs, social world, trajectory, sociability, extimity

Mots clés : blogs, monde social, trajectoire, sociabilité, extimité

Les blogs sont devenus en quelques années l'un des derniers phénomènes incontournables issus du Web. Les chiffres sont a priori éloquents. BlogPulse, moteur de recherche et indicateur de popularité dédié aux blogs, en indexe quelque 126 millions en $2010^{1}$. Derrière ces données se cache une réalité plus contrastée. Sur la centaine de millions, $1,1 \%$ seraient mis à jour au moins une fois par semaine ${ }^{2}$. Lorsque le regard se porte à une échelle micro, sur des blogs qualifiés de manière générale de « blogs extimes ${ }^{3}$ », le maintien

1. «BlogPulse Stats», BlogPulse, http://www.blogpulse.com/, consulté le 7 avril 2010.

2. Francis Pisani, « Blogalaxie/9-133 millions de blogueurs, 1,5 million d'actifs », Transnets, 24 septembre 2008, http://pisani.blog.lemonde.fr/2008/09/24/blogalaxie9-133-millions-deblogueurs-15-million-dactifs/, consulté le 7 avril 2010.

3. Serge Tisseron définit l'extimité comme « le mouvement qui pousse chacun à mettre en avant une partie de sa vie intime, autant physique que psychique. Cette tendance est longtemps passée inaperçue bien qu'elle soit essentielle à l'être humain. Elle consiste dans le désir de communiquer à propos de son monde intérieur » (L'Intimité surexposée, Paris, Ramsay, 2001, p. 52). La pratique du blog " extime » relève de cette logique de publicisation d'éléments intimes et, par-delà, d'une individualisation du tracé des frontières de l'intimité. 
d'une publication sur la durée relève du sacerdoce. Première difficulté, les blogueurs doivent s'assurer à la fois d'être lus, commentés, liés, voir leur présence en ligne confirmée par leurs pairs au risque de perdre la face.

La pratique du blog laisse entrevoir la capacité des utilisateurs à (re)construire leur « micro-monde ${ }^{4} »$ par le biais d'un dispositif de publication en ligne. Autre difficulté, ces mondes disposent de frontières voulues par les pratiquants, mais en définitive peu hermétiques. L'enjeu sous-jacent à l'activité du blogueur peut être formulé ainsi : apparaître aux yeux de ses pairs sans être visible de toute personne extérieure aux réseaux affinitaires. Étant donné le caractère intrinsèquement public de l'activité, ces deux propositions apparaissent a priori antinomiques. L'objet de cet article est de proposer l'exploration d'un territoire interstitiel afin de cerner la manière dont un individu crée et maintient son espace de liberté entre des dispositifs d'écriture contraignants et la présence de lecteurs indésirables.

\section{La construction d'espaces de communication en ligne}

Pour présenter la manière dont ces contraintes sont solutionnées, nous nous appuierons sur l'expérience d'un jeune blogueur. La taille nécessairement restreinte de notre article ne nous permet pas l'exploration de plusieurs trajectoires au risque d'obscurcir la lecture. L'unique cas proposé est jugé représentatif du parcours des blogueurs et permet de cerner certains enjeux liés aux pratiques de publication en ligne.

\section{Détour méthodologique : le suivi à distance des trajectoires}

Auparavant, un rapide détour méthodologique s'impose. Le matériau empirique présenté ci-après a été recueilli au gré du suivi des activités de publication en ligne d'un nombre limité d'individus. Ce recueil repose sur le pari d'un maintien de leur pratique. Leur sélection s'est opérée de manière « naturelle» sur la base d'un corpus de quatre cents unités constitué en $2004^{5}$. Quatre ans plus tard, seuls huit blogueurs identifiés présentaient

4. Nous faisons explicitement référence à l'approche des interactionnistes en termes de mondes sociaux, en particulier à celles de Howard Becker, au travers de Mondes de l'Art (Paris, Flammarion, 1988) et d'Anselm Strauss («Une perspective en termes de monde social », dans Isabelle Baszanger (éd.), La Trame de la négociation. Sociologie qualitative et interactionnisme, Paris, L'Harmattan, 1992, p. 269-282). Par ailleurs, notre lecture de la notion s'inscrit dans la perspective de l'analyse des mondes sociaux par Dominique Pasquier, appliquée aux téléspectateurs des séries adolescentes : «La télévision comme expérience collective : retour sur les Mondes de l'Art », dans Alain Blanc et Alain Pessin (dir.), L'Art du terrain. Mélanges offerts à Howard Becker, Paris, L'Harmattan, 2004, p. 193-218; « La culture comme activité sociale », dans Éric Maigret et Éric Macé (dir.), Penser les médiacultures. Nouvelles pratiques et nouvelles approches de la représentation du monde, Paris, Armand Colin/INA, 2005, p. 103-120.

5. Ce corpus a été constitué dans le cadre d'une observation générale du phénomène à 
une activité de publication manifeste ${ }^{6}$. Celle-ci s'étale sur six années et a comme pivot central le blog, sans toutefois s'y limiter (pages personnelles, forums de discussion, salons IRC). La méthodologie employée s'articule autour de deux étapes nécessaires : la réalisation d'entretiens semi-directifs et d'échanges complémentaires par courriers électroniques sur leur pratique; l'observation du contenu à partir des grilles de lecture issues des entretiens réalisés.

Cette démarche a ouvert la voie à une approche compréhensive. Elle est destinée à saisir la manière dont le recours au blog contribue à la (re)construction des univers propres aux blogueurs. Le blog, entendu comme objet technique, favorise la coopération entre individus et contribue à la formalisation d'un espace à soi sur Internet. La durée offre l'opportunité d'observer les dynamiques de changement liées à leur investissement en ligne, sous les yeux de leurs pairs. Expérimenter un dispositif, gérer de manière ludique et instrumentée une sociabilité sont des pratiques devenues courantes, particulièrement chez les adolescents ${ }^{7}$. Il en va différemment lorsqu'il s'agit de maintenir l'utilisation dans la durée.

\section{Des espaces multiples et articulés les uns aux autres}

Pour cet article, la trajectoire de Rhalph nous servira d'appui empirique. Il s'agit d'un jeune étudiant rennais en informatique qui fait son entrée dans la vie active. Sous ce pseudonyme, il anime depuis octobre 2003 un blog créé à la sortie du lycée. Construit essentiellement autour d'un contenu personnel, son blog est le lieu d'un bricolage identitaire et d'une affirmation de sa subjectivité. Il y relate ses péripéties personnelles et y affiche ses goûts culturels (essentiellement musicaux et littéraires). La construction narrative n'a rien de fictionnel. La publication repose principalement sur la mise en publicité délibérée de tranches de vie et d'états d'âme : vie estudiantine ponctuée par les périodes d'examens, difficultés relationnelles face au sexe opposé. L'empathie exprimée par les commentateurs réguliers du blog contribue à la reconnaissance de sa singularité. Un des traits remarquables de cette trajectoire est la fréquentation assidue d'une des premières plates-formes

l'échelle de la Bretagne, menée par le GIS M@rsouin. Les blogs ont été repérés par le biais des moteurs de recherche et le seul critère discriminant retenu était l'ancrage régional des auteurs. Olivier Trédan, Le Phénomène des blogs, rapport d'étude, Marsouin, 3 juin 2005, http://expert.infini.fr/Le-phenomene-des-blogs, $126 \mathrm{p}$.

6. Une nuance est ici à apporter. L'abandon ou la suppression d'un espace de publication ne signifie pas pour autant l'arrêt d'une activité de publication. La rupture souhaitée dans une trajectoire rend difficile, voire impossible, le suivi des différentes expériences développées par un seul et même individu.

7. Cédric Fluckiger, «La sociabilité juvénile instrumentée. L'appropriation des blogs dans un groupe de collégiens ", Réseaux, $\mathrm{n}^{\circ}$ 138, 2006, p. 109-138; Hélène Delaunay-Téterel, "Sociabilité juvénile et construction de l'identité. L'exemple des blogs adolescents », Informations sociales, $\mathrm{n}^{\circ} 145,2008$, p. 48-57. 
de blogs, $U$-blog ${ }^{8}$, aujourd'hui désactivée. Cet espace a été le lieu d'un engagement fort, reposant sur une série de conventions partagées, dont la mise en récit de soi et un souci accordé à la qualité de l'expression.

Replacé à l'échelle de la trajectoire de Rhalph, le blog ne constitue qu'un espace fréquenté parmi d'autres. Sa présence sur Internet remonte aux débuts des années 2000. Il participait alors assidûment à plusieurs forums de discussion, aux objectifs distincts. Un premier, baptisé Inutile.com, était le lieu de rassemblement de quelques amis du lycée, dont les discussions n'étaient compréhensibles que des seuls membres. Parallèlement, il a développé sa passion pour l'informatique autour d'un logiciel d'édition d'images numériques, Terragen. Ses propres réalisations sont exposées sur un site $w^{2} b^{9}$, la fréquentation des forums est motivée par l'échange de conseils techniques. Par ce biais, il a développé quelques relations affinitaires poussées. Progressivement, il a multiplié les espaces d'intervention à mesure que ceux-ci se sont développés (en 2003, une fiche de présentation sur un site qualifié de social networking, Parano.be; en 2007, un compte sur Facebook, etc.). L'articulation des dispositifs renvoie vers une logique de construction d'un espace de communication sur Internet, déjà identifiée à la fin des années $1990^{10}$.

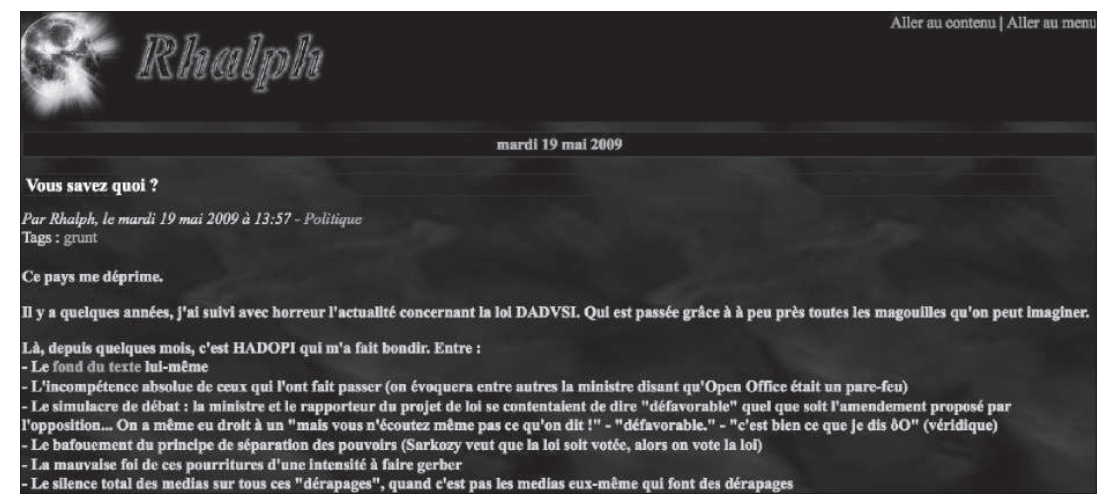

Fig. 1 - Le blog de Rhalph

8. Lancée en novembre 2002, à l'initiative de Stéphane Le Solliec, elle a été vendue à un investisseur, Loïc Le Meur, en octobre 2003, avant d'être cédée en juillet 2004 à la société Six Apart, un des principaux éditeurs de logiciels destinés à la création de blogs. U-blog a été rapidement abandonnée au profit d'un outil plus performant Type Pad. Elle a été désactivée en octobre 2007.

9. Le site est entièrement dédié à « ce logiciel génial qui, pour ceux qui ne le connaissent pas, permet de créer de superbes paysages en quelques clics ». http://terrafgen.free.fr/.

10. Valérie Beaudouin et Julia Velkovska, «Constitution d'un espace de communication sur Internet (Forums, pages personnelles, courrier électronique...) », Réseaux, n 97, 1999, p. 121-177. 


\section{Le blog entre lieux de pratique et construction de rôles sociaux}

Ces éléments posés, il nous faut revenir au blog. La raison est donnée par le blogueur lui-même au détour d'un commentaire.

C'est quand même grâce à $U$-blog que j'ai eu ma première copine (à 20 ans !) ainsi qu'une autre un peu plus tard... Et puis tous ces liens qui se sont créés (Aurora, Falo... Gwenn aussi, elle est quand même devenue ma meilleure amie hein $: P)$ etc ${ }^{11} \ldots$

La solution de blogging U-blog a été le lieu d'un investissement personnel important et occupe une place particulière dans sa trajectoire. Cet espace n'est ni un simple lieu d'affichage, ni déconnecté d'aires de sociabilités parallèles, mais le lieu où s'entrecroisent et s'agrègent des univers distincts. Ce type de parcours permet de mettre en avant deux éléments liés : d'une part, le caractère structurant des dispositifs de publication; de l'autre, la construction de rôles sociaux dans des espaces partagés.

\section{Le fonctionnement d'une plate-forme d'hébergement}

Ce blog, créé en octobre 2003, a été abandonné en mars 2007, Rhalph lui préférant un blog développé sous Dotclear ${ }^{12}$, un logiciel libre d'édition de blogs, et hébergé sur un compte Free ${ }^{13}$. Ce départ n'a pas été sans regret. Dans un billet, il exprime son exaspération :

Après tous les plantages d'U-blog, les multiples attaques de spams, le bug du nombre de commentaires qui a mis deux mois à être corrigé, [...] me voilà maintenant avec [...] à peu près 800 spams à supprimer un à un, avec la lenteur qui caractérise $U$-blog depuis quelques mois... Alors voilà, moi j'en peux plus, je me casse d'ici ${ }^{14}$.

La référence au lieu - ici - et l'attachement ${ }^{15}$ manifesté se réfèrent à une double pratique. La première se centre sur l'activité de publication stricto sensu, que l'on peut qualifier d' « extime ». Elle renvoie en cela à une pratique qui avait cours sur $U$-blog : le suivi à l'écran de subjectivités médiatisées en prenant soin de la qualité de l'écriture. La seconde consiste à parcourir régulièrement les espaces de publication des pairs. La plate-forme $U$-blog a été un espace réticulaire au sein duquel les contributeurs actifs se

11. Anne, «Farewell Ublog», Les mille et une vies, http://www.chiboum.net/index. php?2007/09/13/851-farewell, consulté le 7 avril 2010. Les citations reproduisent l'orthographe et la syntaxe du blogueur.

12. Logiciel libre d'édition de blogs, créé en 2002.

13. http://rhalph.blog.free.fr/.

14. Rhalph, «Plein le cul », http://rhalph.blog.free.fr/index.php?post/2007/03/15/474-pleinle-cul-, consulté le 7 avril 2010.

15. Antoine Hennion, «Une sociologie des attachements. D'une sociologie de la culture à une pragmatique de l'amateur », Sociétés, n 85, 2004, p. 9-24. 
reconnaissaient mutuellement et ont forgé une histoire locale commune. Cette caractéristique est particulièrement visible dans les commentaires postés sur son blog. Ceux-ci sont le fait d'une double population. Parmi ses lecteurs, on retrouve un noyau d'une dizaine d'habitués, avec qui il a noué des relations affinitaires soutenues, les rencontrant régulièrement pour certains, menant de longues sessions de discussion via MSN pour d'autres. À côté de ces proches, plusieurs centaines de blogueurs sont venus réagir à sa publication. Ceux-ci, individus inconnus de l'auteur, ont quasiment tous séjourné sur U-blog. Ces commentaires sont le plus souvent compatissants, fournissant conseils et soutien à l'auteur lors de moments difficiles.

Le passage d'une solution hébergée aux serveurs de Free renseigne sur l'importance du premier dispositif. Du groupe élargi de blogueurs venant commenter ses billets, il ne reste plus que les proches qui disposent de son adresse. Cette transition douloureuse se reflète dans les interventions des lecteurs. Nous avons procédé à la comparaison de deux périodes de publication : avril-juin 2004 et avril-juin 2007. Ces deux périodes marquent, pour l'une, le moment d'un investissement fort au sein de la plate-forme, pour l'autre, le moment où le nouveau blog est créé.

\begin{tabular}{|l|l|}
\hline \multicolumn{1}{|c|}{ Avril-juin 2004 } & \multicolumn{1}{c|}{ Avril-juin 2007} \\
\hline 54 notes publiées & 35 notes publiées \\
\hline 603 commentaires & 172 commentaires \\
\hline 85 blogueurs différents & 35 blogueurs différents \\
\hline 14 ont posté plus de 10 commentaires & 12 ont posté plus de 10 commentaires \\
\hline 54 n'en ont posté qu'un seul & 10 n'en ont posté qu'un seul \\
\hline $\begin{array}{l}\text { Contexte }: \text { forte expansion de } U \text {-blog } \\
(+175 \%)\end{array}$ & $\begin{array}{l}\text { Contexte }: \text { Rhalph a quitté } U \text {-blog le } 24 \\
\text { mars } 2007\end{array}$ \\
\hline
\end{tabular}

Comparaison de l'activité sur le blog de Rhalph

La première période a été marquée par la présence de lecteurs peu réguliers, inconnus de l'auteur, mais présents sur la plate-forme de blogging. Quatorze amis font partie de son réseau relationnel, provenant de plusieurs univers. Deux sont issus d'un forum de discussion autour d'un logiciel d'édition d'images numériques, Terragen; quatre personnes ont noué des liens dans le cadre d'une sociabilité en direct; la majorité - huit - est constituée de blogueurs, n'ayant en commun que la fréquentation d'U-blog. En 2007, la publication s'est faite moins régulière. Le nombre des commentateurs a été divisé par 2,5; celui des commentateurs avec un commentaire unique par cinq. La comparaison de la publication entre ces deux périodes fait état d'un déplacement des thèmes abordés. En 2007, ils étaient polarisés autour d'adresses personnelles à destination de ses lecteurs réguliers et autour de l'actualité politique, liée au déroulé de la campagne présidentielle. En 2004, le contenu était plus personnel. L'auteur y exposait volontiers ses états 
d'âme et multipliait les courts billets dont la seule finalité est de susciter le commentaire à moindre frais.

\section{Des formes d'engagement au sein d'un collectif d'utilisateurs}

Ce que nous voulons souligner au travers de la trajectoire de Rhalph est autant lié à son activité de publication qu'au cadre dans lequel il intervient. En effet, plus qu'un jeune étudiant s'interrogeant sur son avenir, Rhalph a été reconnu comme un des membres actifs s'étant engagé contre l'abandon de la plate-forme et ayant contribué au développement de celle-ci, en proposant quelques outils aux autres utilisateurs. En d'autres termes, la pratique du blog suppose un engagement du pratiquant au sein d'un lieu dans lequel il est connu et reconnu et qui dispose d'une histoire singulière.

L'engagement du blogueur a pris les traits d'une mobilisation, en juillet 2004, destinée à sensibiliser les autres utilisateurs sur l'avenir incertain de la solution. Par exemple, dans une note intitulée « À l'attention de tous les ublogueurs ${ }^{16} »$, il dénonce la cession de la plate-forme à une société éditrice de plates-formes et de logiciels dédiés au blog, Six Apart. Dans ce billet, il expose clairement ses craintes quant à l'avenir d'U-blog. Par ailleurs, il a mis à disposition à maintes reprises ses compétences acquises dans le cadre d'un cursus universitaire, ce qui lui a fourni l'opportunité d'assumer un rôle de développeur amateur reconnu par ses pairs. Ainsi, il a proposé en 2005 un logiciel destiné à la transformation de saisie textuelle en mise en forme en code html, fonctionnant sous le navigateur Firefox. « Vous êtes passé sous Firefox et vous ne pouvez plus blogger parce que vous ne savez pas comment vous servir de l'html, ou vous trouvez que taper vos notes en html prend trop de temps? Ce logiciel est fait pour vous ${ }^{17}$. \ Les réactions postées sont alors du registre du remerciement : trente-neuf commentaires ont été laissés, comptant parmi les notes les plus commentées. Parmi les intervenants, on retrouve ses lecteurs réguliers, mais aussi onze blogueurs qui viennent saluer l'initiative : «Salut Rhalph! Je compte passer sur Firefox, mais le dernier truc qui me retient c'est bien le problème de posts sur u-blog! » Une attention similaire se retrouve quelques mois avant la fermeture de la plate-forme. « J'ai terminé le programme pour exporter n'importe quel blog U-blog [...] vers Dotclear ${ }^{18}$. \ Ce programme a ainsi permis aux U-blogueurs de pouvoir conserver leurs archives en dépit de l'imminence de la fermeture annoncée de la plate-forme.

16. Rhalph, « ̀̀ l'attention de tous les u-blogueurs », http://rhalph.blog.free.fr/index. php?post/2004/07/19/197-a-l-attention-de-tous-les-u-bloggueurs, consulté le 7 avril 2010.

17. Rhalph, «Logiciel gratuit pour blogguer avec Firefox », http://rhalph.blog.free.fr/index. php?post/2005/07/04/300-logiciel-gratuit-pour-blogger-avec-firefox-, consulté le 7 avril 2010. 18. Rhalph, « Fuite du navire », http://rhalph.blog.free.fr/index.php?post/2007/03/24/477fuite-du-navire, consulté le 7 avril 2010. 
Cette reconnaissance est la source d'un attachement à ce lieu. Des subjectivités se sont mises en scène et se sont vu reconnaître mutuellement. La plate-forme est un lieu habité, où les individus ont placé une charge émotionnelle : ils sont attachés au dispositif, à son visuel, à ses fonctionnalités.

\section{Gérer ses apparitions sur la durée}

Le parcours d'un blogueur tel que présenté jusqu'à maintenant laisse entrevoir l'importance de la réflexivité qui se traduit par un attachement aux objets de la pratique et un engagement au travers de ceux-ci. Néanmoins, cette présentation demeure incomplète dans la mesure où n'apparaissent pas les tensions générées par 1'articulation de cet espace aux autres contextes dans lesquels évolue un individu.

\section{Le constat d'un cadre d'apparition perméable}

Une récurrence apparaît dans le parcours des blogueurs : le recours à un pseudonyme. Si de prime abord, il s'agit d'un truisme, il permet de pointer le souci accordé à la préservation d'une relative autonomie à l'égard des autres cadres dans lesquels les individus sont amenés à intervenir (famille, travail, etc.). La raison de l'abandon de son espace de publication peut être attribuée à la découverte que des proches non ratifiés sont lecteurs assidus en dépit de ses efforts pour que l'espace de publication reste cantonné au seul cadre des interactions entre pairs. La gestion de cette tension, c'est-à-dire la capacité à n'être lu que par un public déterminé, apparaît comme un élément explicatif du maintien d'une activité de publication sur un temps relativement long.

Pour Rhalph, cette tension se manifeste directement sous forme d'adresses à ses lecteurs.

\footnotetext{
Depuis quelque temps, je commence à être obnubilé par l'anonymat de mon blog. Je crois que le déclencheur, ça a été quand mon ancienne maîtresse de stage est tombée sur mon blog... Sachant que j'avais fait plusieurs posts dans lesquels je disais que je foutais rien en stage (même si au final j'ai terminé ce que j'avais à y faire). [...] J'étale beaucoup ma vie privée ici, et je n'ai pas envie que ça me porte préjudice si un indésirable tombe dessus ${ }^{19}$.
}

Ce billet fait suite à une mesure extrême mise en œuvre. Il a entrepris de désindexer son blog de Google et renvoie les lecteurs issus du moteur de recherche vers un faux blog annonçant la suspension de son activité ${ }^{20}$. Quelques semaines plus tard, il exprime un sentiment qui apparaît contradictoire.

19. Rhalph, « Ce blog a quatre ans », http://rhalph.blog.free.fr/index.php?post/2007/10/19/ Ce-blog-a-quatre-ans, consulté le 7 avril 2010.

20. «Stop. J'arrête ce blog, marre, j'y vois plus d'intérêt. Les gens qui le veulent pourront me contacter sur Msn. », http://rhalph.canablog.com, consulté le 24 novembre 2008. 
Bon, Dotclear c'est génial, [...] je me sens complètement chez moi. Seulement, en mettant mon blog sur un site perso, je me suis un peu exclu de la blogosphère. Ce que j'adorais chez U-blog c'était de faire partie d'une communauté, apparaitre en page d'accueil... Vraiment l'aspect communautaire, quoi. [...] Ça m'emmerde un peu qu'il n'y ait jamais de nouvelle personne à lire ce blog 21 .

Ces propos tenus révèlent deux caractéristiques intrinsèques des mondes sociaux. D'une part, ils sont segmentés et entrecroisés. Dans la mesure où les individus interviennent sur de multiples scènes, les mondes sociaux sont amenés à s'interpénétrer. De ce fait, ils ne disposent pas de frontières stables et hermétiques. D'autre part, ils sont des processus et sont de ce fait dans une dynamique permanente d'expansion, sous peine de disparition. Ramenée à la pratique du blog, cette double contrainte fait des blogueurs des entrepreneurs de leur propre notoriété. Cette caractéristique implique un renouvellement de leur lectorat, sciemment choisi ou du moins accepté.

\section{L'impératif d'un renouvellement de son lectorat}

Cette tension entre un souci de ne pas apparaître aux yeux de tous et celui d'agréger de nouveaux lecteurs n'est qu'en apparence insoluble. Elle ne l'est du moins que si notre regard ne se porte que sur la seule pratique du blog. Or, les trajectoires d'usage des blogueurs manifestent une capacité à multiplier les espaces d'intervention en les articulant à leur blog. Tirons de l'expérience de Rhalph un exemple significatif. Fin 2007 disparait la plateforme à vocation communautaire sur laquelle il évoluait depuis quatre ans. Parallèlement, il prend conscience de conséquences de sa publication qu'il juge potentiellement néfastes, en particulier dans la perspective d'une entrée prochaine sur le marché du travail. Toutefois, il occupe d'autres espaces

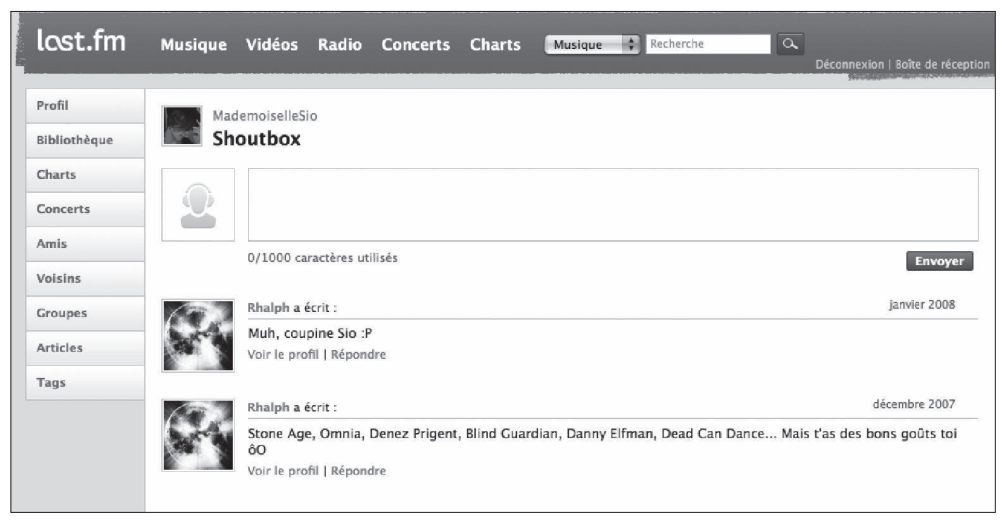

Fig. 2 - Dépôt de deux commentaires sur un profil sur Last.fm

21. Rhalph, « Dilemme (lemme) », http://rhalph.blog.free.fr/index.php?post/2008/01/18/ Dilemme-lemme, consulté le 7 avril 2010. 
collectifs. Parmi ceux-ci, il fréquente régulièrement le site communautaire d'écoute de musique en ligne last.fm ${ }^{22}$. Sur sa fiche, il se présente au travers de ses préférences culturelles : musique gothique et littérature fantastique. La consultation des profils des « voisins » permet d'identifier les individus aux goûts similaires : parmi ceux-ci une fan de musique celtique.

Via ce site, j'ai découvert un nouveau blog. J'ai vu sur le profil de Gwenn [sa meilleure amie] que quelqu'un l'avait commenté. Et je suis allé voir son profil. C'est là que je me suis rendu compte qu'on avait plein de goûts en commun ${ }^{23}$. J'avais laissé un commentaire, parce que je voulais en savoir plus sur elle. J'ai laissé l'adresse de mon blog et mon adresse MSN. Et elle m'a contacté quelques jours après.

Les premiers contacts ont été noués non pas sur Last.fm, mais sur leurs blogs respectifs via l'échange de commentaires. Ceux-ci ne sont déposés, dans un premier temps, que sous les billets faisant référence aux goûts culturels communs; les billets plus personnels restent l'apanage des lecteurs assidus. Progressivement, les échanges se font plus réguliers. Ils ne concernent plus seulement les billets parlant de musique ou de littérature fantastique, mais des billets plus personnels. Parallèlement, une complicité naît via MSN. Au bout de quelques mois d'échanges, les deux blogueurs se rencontrent à plusieurs reprises et se commentent régulièrement l'un l'autre.

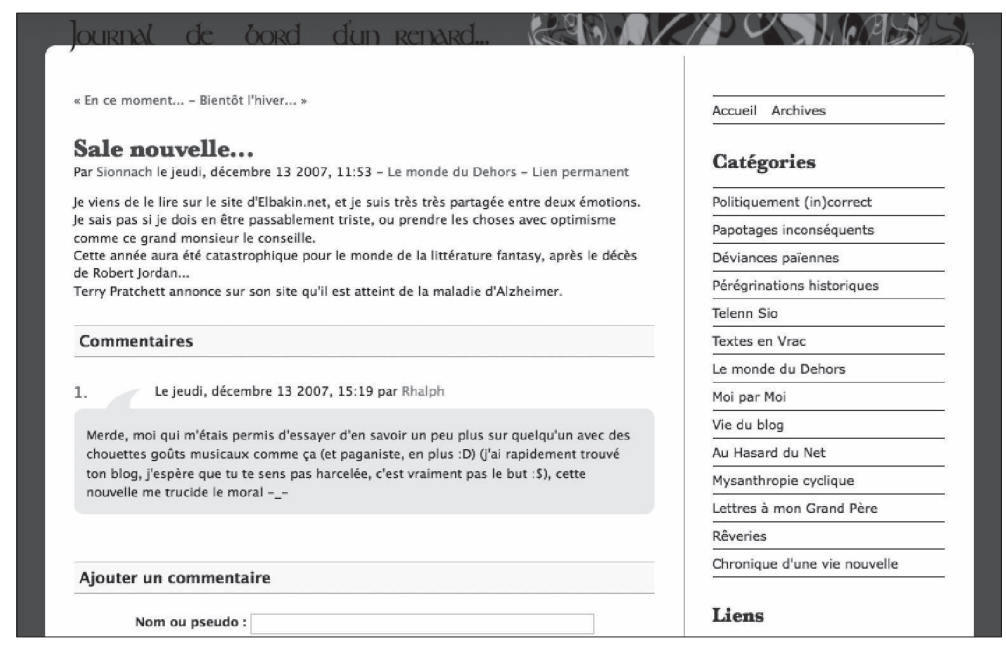

Fig. 3 - Premier commentaire de Rhalph déposé sur le blog de Sionnac

22. Last.fm est l'une des méta-radios les plus populaires du Web, rachetée en mai 2007 par $C B S$. Il s'agit d'un site web précurseur dans l'écoute légale de la musique en ligne. Ayant une vocation communautaire, il propose une médiation permettant l'affichage des « voisins » musicaux, c'est-à-dire ceux présentant le plus d'affinité culturelle.

23. Il a pu confirmer cette proximité pour la consultation de son blog personnel, http://sionnach.tarlak.net/, via son profil de Last.fm, http://www.lastfm.fr/user/MademoiselleSio/. 
Cet exemple ne doit pas laisser entendre qu'il n'existe qu'une modalité de renouvellement de son lectorat, passant inévitablement par Internet. Les rencontres effectuées en direct constituent également un réservoir potentiel. Ce cas nous montre les tactiques mises en œuvre par les blogueurs. L'utilisation d'un dispositif d'écoute de musique en ligne devient une entrée pour recruter des lecteurs que l'on souhaite fidéliser. Loin d'être dans un espace ouvert, s'exprimant face à un autrui généralisé, les blogueurs prennent le soin d'entretenir et de renouveler leur lectorat, condition nécessaire pour éviter le déclin progressif de leur monde en ligne. Dans cette perspective, le recours à l'ensemble des médiations offertes par les dispositifs (sites parcourus, espaces des commentaires) est incontournable pour générer du lien social.

\section{Conclusion}

À travers la trajectoire d'un blogueur, nous avons tenté de présenter la manière dont se construisent les mondes sociaux en ligne. Leur édification requiert à la fois, de la part des individus, un attachement à l'objet de leur pratique et un engagement aux yeux des pairs. Les situations d'interaction concourent à la production de rôles sociaux singuliers, propres à ces espaces. Par effet de miroir, il est possible d'expliquer partiellement l'abandon d'un espace de publication : un dispositif qui ne répond plus aux attentes assignées par un utilisateur, la présence trop encombrante de lecteurs non désirés, l'absence de pairs venant confirmer par leurs interventions répétées la singularité du blogueur. Toutefois, aucune de ces raisons ne peut signifier la fin d'une activité. Celle-ci peut potentiellement être déplacée, se dérouler dans d'autres lieux, avoir des finalités différentes. Il convient donc de distinguer le blog comme résultat d'une pratique de publication et la pratique elle-même.

Proposer une grille de lecture en termes de monde social pour décrypter cette activité s'avère pertinent. Outre le fait qu'elle invite à la décrire comme un processus et une dynamique permanente, elle permet de penser le blogueur comme inséré dans des réseaux de coopération où s'élaborent des conventions communes. En premier lieu, l'échange de commentaires contribue au renfort mutuel de leurs micro-mondes. Autre élément, la conception de leur public se limite souvent à leurs pairs et non à tous les lecteurs potentiels. L'activité du blogueur semble alors se résumer à une capacité à maximiser ses apparitions auprès de son réseau, tout en les limitant vis-àvis de l'extérieur.

Olivier Trédan

Université de Rennes 1 - CRAPE 